Sādhanā Vol. 39, Part 4, August 2014, pp. 921-937. (C) Indian Academy of Sciences

\title{
Electrocardiogram de-noising based on forward wavelet transform translation invariant application in bionic wavelet domain
}

\author{
MOURAD TALBI
}

High Institute of Applied Mathematics and Informatics of Kairouan, University of Kairouan, Kairouan, Tunisia

e-mail: mouradtalbi196@yahoo.fr

MS received 9 December 2012; revised 15 December 2013; accepted 24 January 2014

\begin{abstract}
In this paper, we propose a new technique of Electrocardiogram (ECG) signal de-noising based on thresholding of the coefficients obtained from the application of the Forward Wavelet Transform Translation Invariant (FWT_TI) to each Bionic Wavelet coefficient. The De-noise De-noised ECG is obtained from the application of the inverse of $B W T\left(B W T^{-1}\right)$ to the de-noise de-noised bionic wavelet coefficients. For evaluating this new proposed de-noising technique, we have compared it to a thresholding technique in the FWT_TI domain. Preliminary tests of the application of the two de-noising techniques were constructed on a number of ECG signals taken from MIT-BIH database. The obtained results from Signal to Noise Ratio (SNR) and Mean Square Error (MSE) computations showed that our proposed de-noising technique outperforms the second technique. We have also compared the proposed technique to the thresholding technique in the bionic wavelet domain and this comparison was performed by SNR improvement computing. The obtained results from this evaluation showed that the proposed technique also outperforms the de-noising technique based on bionic wavelet coefficients thresholding.
\end{abstract}

Keywords. Bionic wavelet transform (BWT); forward wavelet transform translation invariant (FWT_TI); de-noising; thresholding; ECG signal.

\section{Introduction}

The ECG signal is a biomedical signal and is widely studied and applied in clinic. A normal ECG waveform is typically composed of QRS complexes, T wave and P wave. So the accuracy of them is very important for ECG analysis. Although, due to faintness of the ECG signal, it 
is very probable to be corrupted by different types of noise while recording and gathering. The efficient suppression of those noises is usually a main problem in determination of ECG characteristics. Lately, wavelet transform has been extensively employed in signal processing and this because of characteristics of time-frequency localization (Addison 2005). Two principal types of wavelet de-noising technique were used for ECG de-noising. The first is wavelet transform modulus maximum technique. This technique permits to cancel noises and retain the information of the original signal in maximum at the same time, but the computation amount is huge and the computation process may be unstable (Sahambi et al 1998; Han et al 2009). The second denoising technique is based on the wavelet threshold. It consists of selecting a threshold for the wavelet coefficients in advance. The discrete wavelet coefficients at different scales are obtained by applying the Discrete Wavelet Transform (DWT) to the noisy signal (Mallat \& Zhong 1991; Gao et al 2003; Yao \& Zhang 1999). Normally, the discrete wavelet coefficients, having magnitudes smaller than the predetermined threshold, are caused by the noise and replaced by zero, and the remaining coefficients having magnitudes larger than the predetermined threshold are caused mainly by the signal and they are conserved (Hard thresholding case) or shrunk (Soft thresholding case) (Sayadi \& Shamsollahi 2006). The de-noise de-noised signal is then reconstructed from the threshold of discrete wavelet coefficients by applying the inverse of the discrete wavelet transform (IDWT) to these coefficients. This technique is simple and easy and to be used for ECG de-noising. Though the hard thresholding may lead to the reconstructed ECG signal oscillation and the soft thresholding de-noising technique may reduce the ECG waveforms amplitudes, and especially reduce the amplitudes of the $\mathrm{R}$ waves (Han et al 2009). In this paper, we propose a new de-noising technique based on the de-noising of the noisy bionic wavelet coefficients obtained from the application of the Bionic Wavelet Transform (BWT) to the noisy ECG signal. The de-noising of each noisy bionic wavelet coefficient is performed by the thresholding of the coefficients obtained from the application of the FWT_TI to that coefficient.

\section{The wavelet transform (WT)}

Wavelet analysis is used for transforming the signal under investigation into joined temporal and spectral representation (Addison 2005; Janusek et al 2011) which permits us to ameliorate signal analysis and processing. The wavelet transform can be represented as an application of low-pass and high-pass filters. These two filters are with cut-off frequency at the middle of the sampling frequency (Janusek et al 2011). Extending 1D-adjustable-resolution to 2D-adjustable-resolution for better trade off both time and frequency resolutions has been a topic in WT for a long time (Sayadi \& Shamsollahi 2006). Many attempts concentrate on the wavelet transform application to the signal. Research has proven that the mother wavelet function choice severely affects the quality and appearance of the resultant time-frequency representation (Sayadi \& Shamsollahi 2006). In this case, efforts have been made in order to introduce adaptation into WT. AWT applications in bio-signal processing, however, are limited and this due to the facts that the AWT computation cost is typically very high and there is no entropy criterion suitable for biomedical applications (Sayadi \& Shamsollahi 2006). Among those techniques, the BWT, initially introduced by Yao \& Zhang (2000), is principally developed and being optimized by the human bio-system and has showed very good results in speech processing. For this reason, we have introduced the BWT in our proposed ECG de-noising system. 


\section{The bionic wavelet transform (BWT)}

Formerly, Yao adopted a nonlinear auditory model (Yao \& Zhang 2000) in which each basilar membrane point is modelled by the following equation:

$$
\ddot{d}(x, t)+\frac{R_{e q}(x, d) \cdot d(x, t)}{L(x)}+\omega_{0}^{2}(x) \cdot d(x, t)=P,
$$

where, we have:

$$
R_{e q}=R(x)-G_{1}(x) \frac{d 1 / 2}{d 1 / 2+|d(x, t)|} R(x) .
$$

According to Eq. (1), it is clear that a basilar membrane point can be modelled by a BPF which has a nonlinear damping (Sayadi \& Shamsollahi 2006). Moreover, it has been proved that a nonlinear compliance is also needed. This way the resulting the factor of quality is expressed as:

$$
Q_{e q}=R_{e q}^{-1} \sqrt{L(x) / C_{e q}(x)}
$$

It is sufficient to replace the constant $Q_{0}$ of the wavelet transform (WT) by a variable $Q_{T}$ in order to introduce this active mechanism to this transform (Yao \& Zhang 2000). In effect, the passage from WT to the bionic wavelet transform (BWT) is performed by the use of a T-function adopted from the ear model (Sayadi \& Shamsollahi 2006). Hence, the adaptation of the BWT is insured by the T-function. The relation between quality factors for the ear model is given by the following equation:

$$
Q_{e q}=\left(1-G_{2} \frac{d_{1 / 2}}{d_{1 / 2}+|d|}\right)^{-1}\left(1+G_{2}|\partial d / \partial t|\right)^{-1} \text {. }
$$

To replace the constant quality factor $Q_{0}$ with $Q_{T}$, changes can be made in the mother wavelet function $h(t)$. The admissible condition for this mother wavelet implies that it has some oscillations. Those oscillations can be expressed as follow:

$$
h_{T}(t)=\frac{1}{T \cdot \sqrt{a}} \tilde{h}\left(\frac{t}{T}\right) \cdot \exp \left(j 2 \pi f_{0} t\right),
$$

where $f_{0}$ represents the centre frequency of the mother wavelet $h(t)$ and $\tilde{h}(t)$ is its envelope function. Consequently, the BWT mother function can be expressed by the Eq. (5). Therefore, with the new variable mother wavelet, the BWT of a signal $x(t)$ is defined as follow:

$$
\begin{aligned}
B W T_{x}(\tau, a) & =\int x(t) h_{T}^{*}\left(\frac{t-\tau}{a T}\right) d t \\
& =\frac{1}{T \sqrt{a}} \int x(t) \cdot \tilde{h}^{*}\left(\frac{t-\tau}{a \cdot T}\right) \cdot \exp \left(-j 2 \pi f_{0}\left(\frac{t-\tau}{a}\right)\right) d t .
\end{aligned}
$$

We can find the relation between quality factors of WT and BWT by simply looking at the Fourier transform of their mother wavelet functions that is simply stated as:

$$
Q_{T}=T Q_{0}
$$


By comparing this relation with eq. (4), then the T-function is expressed as:

$$
\begin{aligned}
T(\tau+\Delta \tau)= & \left(1-\tilde{G}_{1} \frac{B W T_{s}}{B W T_{s}+\left|B W T_{X}(\tau, a)\right|}\right)^{-1}, \\
& \times\left(1+\tilde{G}_{2}\left|\partial B W T_{x}(\tau, a) / \partial t\right|\right)^{-1}
\end{aligned}
$$

where $B W T_{s}, \tilde{G}_{1}$ and $\tilde{G}_{2}$ are constants and $B W T_{x}(\tau, a)$ represents the BWT coefficient at time $\tau$ and scale $a$ and $\Delta \tau$ designates the calculation step.

When the signal and its first-order differential are continuous then the original signal can be reconstructed without distortion by $B W T^{-1}$ (Yao \& Zhang 2001).

\section{New proposed de-noising technique}

As mentioned previously we propose in this paper, a new ECG de-noising technique based on thresholding of the coefficients obtained from the application of the forward wavelet transform translation invariant (FWT_TI) in Bionic Wavelet Transform (BWT) domain. This proposed denoising technique is summarised by the block diagram given in figure 1. As shown in this figure, we first apply the bionic wavelet transform (BWT) to the noisy ECG signal. The application of the BWT is performed by using 30 scales (Mourad et al 2010) instead of 22 scales (Yao \& Zhang 2001; Sayadi \& Shamsollahi 2006).

For an ECG signal, the most important feature is the frequency range in which its main components occur (Sayadi \& Shamsollahi 2006). Despite the existence of some other components like VLPs, we are interested in this paper in P, Q, R, S and T waves such as in the reference (Sayadi \& Shamsollahi 2006).

In references Yuan (2003), Johnson et al (2007), the value of $f_{0}$ (the initial centre frequency of the mother wavelet) is equal to $15165.4 \mathrm{~Hz}$. As the scale increases, the centre frequency goes smaller and smaller in the following way (Sayadi \& Shamsollahi 2006):

$$
f_{m}=f_{0} / q^{m}, q>1, m=1,2, \ldots
$$

We don't need such a high frequency $f_{0}$ for the ECG signal. Sayadi \& Shamsollahi (2006) have optimized the value of $f_{0}$ by running the program for different values of $f_{0}$ and then minimizing the gradient of error variance by comparing the results numerically and morphologically with each other. It has been found that if $f_{0}$ belongs to the range of 360 to $500 \mathrm{~Hz}$ there would be no much distortion on the analyzed ECG signal (Sayadi \& Shamsollahi 2006). In their work, Sayadi \& Shamsollahi (2006) have chosen $400 \mathrm{~Hz}$ as the value of $f_{0}$ and in our work; we have chosen the same value for $f_{0}$ as in Sayadi \& Shamsollahi (2006). We have also chosen the value $q=1.1623$ such as in the reference Yuan (2003) \& Johnson et al (2007). Other parameters are those selected in the references (Yuan 2003; Johnson et al 2007) and they are the following quantities: $B W T_{s}=0.8, \tilde{G}_{1}=0.87$ and $\tilde{G}_{2}=45$. In this work, the computation step is also equals to $\Delta \tau=1 / f_{s}$ where $f_{s}$ be the sampling frequency. As shown in the block diagram in figure 1, the second step of the proposed de-noising technique consists in applying the forward wavelet transform translation invariant (FWT_TI) to each bionic wavelet coefficient $w_{i}, 1 \leq$ $i \leq 30$, then each coefficient obtained from this decomposition is thresholded and the inverse of $F W T \_T I, I W T \_T I$, is applied to the threshold of coefficients in order to obtain one denoise de-noised bionic wavelet coefficient. Finally, the inverse of BWT, $B W T^{-1}$, is applied to the thirty de-noise de-noised bionic wavelet coefficients $\hat{w}_{i}, 1 \leq i \leq 30$ in order to obtain the de-noise de-noised ECG signal. 


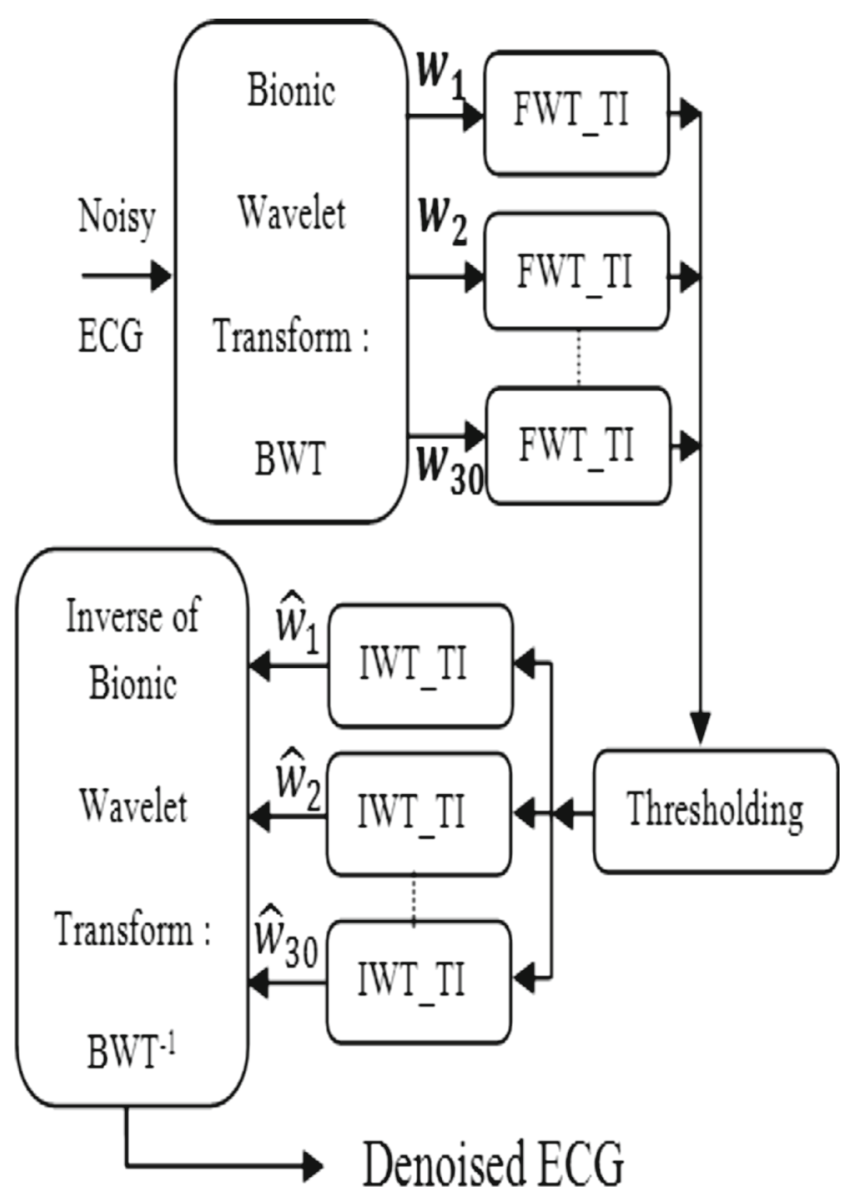

Figure 1. The block diagram of the proposed method.

Our proposed ECG de-noising technique in this work, was previously applied in Mourad et al (2012) to a number of ECG signals taken from MIT-BIH database in order to smooth them and make easier the detection of the different ECG peaks especially the P and T waves. The use of the proposed technique in Mourad et al (2012) was not performed on noisy ECG signals but just for pre-processing in order to facilitate the different peaks detection of the ECG signal. However, in this paper, we have treated the problem of cancelling white Gaussian noise corrupting the ECG signal at different SNRs, by applying our approach.

\section{Translation invariant de-noising}

De-noising with the classical (orthogonal, maximally-decimated) wavelet transform sometimes exhibits visual artefacts such as Gibbs phenomena in discontinuities neighbourhood of discontinuities and this due to the lack of translation invariance of the wavelet basis (Coifman \& Donoho 1995). One technique for suppressing such artefacts, termed 'cycle spinning' by Coifman, consists in 'averaging out' the translation dependence (Coifman \& Donoho 1995). For a range of shifts, the data is shifted (left or right as the case may be), de-noise the shifted data, and then 
the de-noised data is unshifted. This is done for each of a range of shifts, and averaging the several results so obtained, gives a reconstruction subject to far weaker Gibbs phenomena than thresholding based de-noising employing the classical orthogonal wavelet transform (Coifman \& Donoho 1995).

For de-noising in Wavelet Packet domain, which consists in Fourier Transform segmentation of the signal employing a recursive dyadic grid, series de-noising can exhibit mirror symmetries across segmentation points in the frequency domain. In case that the signal is acoustic information, spurious musical notes are generated by these artificial symmetries. Those notes are distracting and have nothing to do with the actual sounds in the original signal (Coifman \& Donoho 1995).

\subsection{Shifts in time and frequency}

The artefacts previously mentioned, are all connected in some way with the precise alignments between features in the signal and features of basis elements; signals exhibit similar features but with slightly different alignment in time or frequency might generate fewer of the artefacts (Coifman \& Donoho 1995). For Haar basis, no real problems are caused by discontinuities at $n / 2$ (Coifman \& Donoho 1995). In wavelet packet analysis, signals with no frequency content near segmentation points in the frequency domain will generate few artefacts in wavelet packet de-noising (Coifman \& Donoho 1995).

One method to correct unfortunate miss-alignments between features in a basis and features in the signal consists in forcibly shifting signals so that their features change positions. The hope is that the shifted signal analysis does not exhibit the undesirable artefacts, and that this analysis can later be unshifted. Coifman \& Donoho (1995) have introduced two operators, of time- and frequency- shift respectively. For a signal $\left(x_{t}, 0 \leq t<n\right)$, we let $S_{h}$ designates the 'circulant shift' by $h,\left(S_{h} x\right)_{t}=x_{(t+h) \bmod n}$ and $M_{\zeta}$ designates the modulation by $\zeta$ so we have $\left(M_{\zeta} x\right)_{t}=e^{i \zeta t} x_{t}$. These two operators are unitary and therefore invertible:

$$
\begin{gathered}
\left(S_{h}\right)^{-1}=S_{-h} . \\
\left(M_{\zeta}\right)^{-1}=M_{-\zeta} .
\end{gathered}
$$

In terms of these operators, the idea of shifting for avoiding artefacts is summarized as follow: let $T$ is an analysis technique then calculate the time-shifted version which is (Coifman \& Donoho 1995):

$$
\tilde{T}\left(x ; S_{h}\right)=S_{-h}\left(T\left(S_{h}(x)\right)\right) .
$$

On the other hand, the frequency-shifted version is:

$$
\tilde{T}\left(x ; M_{\zeta}\right)=M_{-\zeta}\left(T\left(M_{\zeta}(x)\right)\right) .
$$

The missing ingredient consists of knowing the 'correct' shift parameter choice $h$ or $\zeta$. One sensible method for selecting such a parameter lies in optimization: develop a quantitative measure of the 'well-alignment' of the signal, selecting a best value of the shift parameter $h$ or $\zeta$.

\subsection{Averaging shifts}

It may be well that a given signal can be re-aligned for minimizing artefacts; however there is no guarantee that this will usually be the case. When several discontinuities are contained in a signal, these may interfere with each other: the best shift for one discontinuity in the signal may 
also be the worst shift for another discontinuity. Therefore, Coifman \& Donoho (1995) have abandoned the hope of locating a single shift, ideal for aligning all features of the signal, and tried instead another approach, which consists in applying a range of shifts, and average over the different results so obtained. For time shifts this means that a shifts range $H$ is considered so we have as follow:

$$
\bar{T}\left(x ;\left(S_{h}\right)_{h \in H}\right)=\text { Ave }_{h \in H} S_{-h}\left(T\left(S_{h}(x)\right)\right) .
$$

Or, in other words:

$$
\text { Average[Shift - DeNoise - Unshift }] \text {. }
$$

For frequency shifts, this means that a range of modulations $X$ was considered as follow:

$$
\bar{T}\left(x ;\left(M_{\zeta}\right)_{\zeta \in X}\right)=A v e_{\zeta \in X} M_{-\zeta}\left(T\left(M_{\zeta}(x)\right)\right) .
$$

Or in other words:

$$
\text { Aver[Modulate - De - noise - Demodulate]. }
$$

One of Coifman et al (Berger et al 1994; Saito 1994) has been experimenting with ideas of this kind in applied work; he has named this 'Cycle Spinning'. Coifman \& Donoho (1995) have applied Cycle-Spinning Wavelet Shrinkage on a number of noisy signals and the obtained results showed that pseudo-Gibbs oscillations are considerably reduced.

\subsection{Fully TI de-noising}

A complete statement of the algorithm of for $T_{\eta}$ is as follows. We suppose that the variables ncol and $t$ have been appropriately initialized, and then execute the code (Coifman \& Donoho 1995):

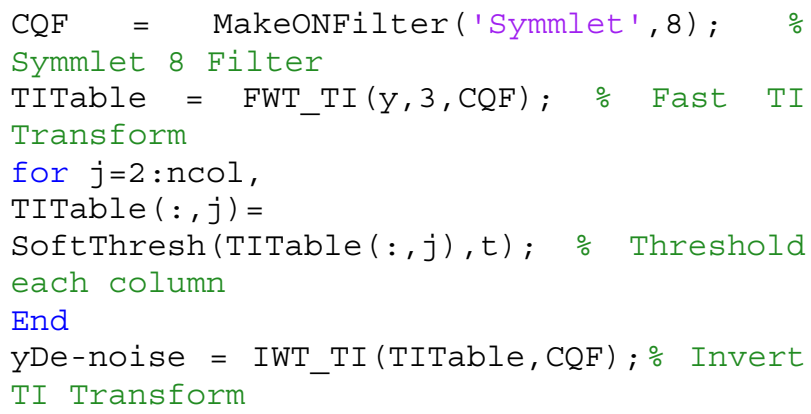

The key computations here are performed by FWT_TI and IWT_TI which rapidly go from signal domain to TI table and back.

\subsection{TI table}

Let $x$ be a vector having a dyadic length $n=2^{J}$, and let $\tau(x)$ be the corresponding TI Table. This has three key properties: (i) for any integer $h$, the wavelet coefficients of the circulant shift $W\left[S_{h} x\right]$, takes order $n$ time. The TI Table is $n$ by $D$ array, where $0 \leq D \leq \log _{2}(n)$. The table formally has the same structure as a wavelet packet table or cosine packet table. The d-th column has $n$ entries partitioned into $2^{d}$ 'boxes', each box with $n / 2^{d}$ entries (Coifman \& Donoho 1995). The interpretation of the 'boxes', they correspond to the $2^{d}$ different collections of wavelet coefficients that can occur in the wavelet expansion at level $J-d$ under different 
shifts of the input vector $x$. The TI table is calculated by a series of decimation and filtering operations, just like the wavelet transform, only an extra-element - a circulant shift - is thrown in. Let $G$ and $H$ stand for the usual down-sampling high pass and low pass operations of wavelet theory, let $S_{h}$ again stand for circulant shift by $h$,and set $\beta_{J, 0}=s$. Then put (Coifman \& Donoho 1995):

$$
\alpha_{J-1,0}=G S_{0} \beta_{J, 0} ; \quad \alpha_{J-1,1}=G S_{1} \beta_{J, 0},
$$

and

$$
\beta_{J-1,0}=H S_{0} \beta_{J, 0} ; \quad \beta_{J-1,1}=H S_{1} \beta_{J, 0} .
$$

Continue recursively:

$$
\alpha_{j, 2 k}=G S_{0} \beta_{j+1, k} ; \quad \beta_{j, 2 k+1}=H S_{1} \beta_{j+1, k},
$$

and so on.

To fill out the TI table, place, in box $k$ of column $d$, the vector $\alpha_{J-d, k}$; in an extra column, place all the $\beta_{j, k}^{\prime}$ s computed at the final stage (we use the zero-th column for this).

To state formally the invariance property owned by TI tables, assume $\tau(x)$ be the TI Table corresponding to $x$ and $\tau\left(S_{h} x\right)$ be the table corresponding to the circulant shift $S_{h} x$. Therefore, for each shift $h \in\{0, \ldots, n-1\}$ there is a permutation of matrix entries $\Pi_{h}$ so that:

$$
\Pi_{h} \tau(x)=\tau\left(S_{h} x\right) .
$$

The traditional wavelet transform consists of the un-shifted data:

$$
W[x]=\left(\beta_{j_{0}, 0}, \alpha_{j_{0}, 0}, \alpha_{j_{0}+1,0}, \ldots, \alpha_{J-1,0}\right) .
$$

Since the right side of $(21), \tau\left(S_{h} x\right)$ contained all the coefficients obtained from the application of the wavelet transform to the shift $S_{h} x$, therefore, those same wavelet coefficients are already present in the TI Table $\tau(x)$; they just require to be correctly unpacked (Coifman \& Donoho 1995).

More exactly, the wavelet transform of a circulant shift of $x$ is performed as follow (Coifman \& Donoho 1995):

$$
W\left[S_{h} x\right]=\left(\beta_{j_{0}, k_{j_{0}}}, \alpha_{j_{0}, k_{j_{0}}}, \ldots, \alpha_{j_{0}+1, k_{j_{0}+1}}, \ldots, \alpha_{J-1, k_{J-1}}\right) .
$$

For an adequate sequence $\left(k_{j 0}, k_{j 0+1}, \ldots, k_{J-1}\right)$, where we have $k_{j}=2 k_{j+1}+b_{j}$ and $b_{j} \in\{0,1\}$. The bits $b_{j}$ encode the shift $h$ in a special binary notation.

For extracting the wavelet transform of a circulant shift from a TI table, specifying the shift is therefore required and this in terms of a special coding of $h$.

\subsection{Inversion}

The problem is how to go back from a TI table to the original signal, the solution is to systematically average: start with $j=J-D$ and then for each $k$ in the range $0 \leq k \leq 2^{j}$, compute (with the help of the usual up-sampling operators $G^{*}$ and $H^{*}$ ):

$$
\begin{aligned}
& \gamma_{k}=\left(S_{0} G^{*} \beta_{j, 2 k}+S_{-1} G^{*} \beta_{j, 2 k+1}\right) / 2, \\
& \delta_{k}=\left(S_{0} H^{*} \alpha_{j, 2 k}+S_{-1} H^{*} \alpha_{j, 2 k+1}\right) / 2 .
\end{aligned}
$$


And we have:

$$
\beta_{j+1, k}=\gamma_{k}+\delta_{k}
$$

After exhausting all $k$ at one level, set $j=j+1$ and repeat. After reaching $j=J$, stop. Set $\tilde{s}=\beta_{J, 0}$.

When applied to the TI table generated from signal $s$, the result is $\tilde{s}=s$. When applied to a thresholded TI table, the result is an average of all $\mathrm{n}$ reconstructions one from an un-shifted series, one from a shifted one - is responsible for this result (Coifman \& Donoho 1995).

\section{Translation invariant de-noising in bionic wavelet domain}

Many signal de-noising systems based on wavelets use other tools. Among these systems, we can mention the ECG de-noising systems using Wiener filtering (Nikolaev \& Gotchev 2000;

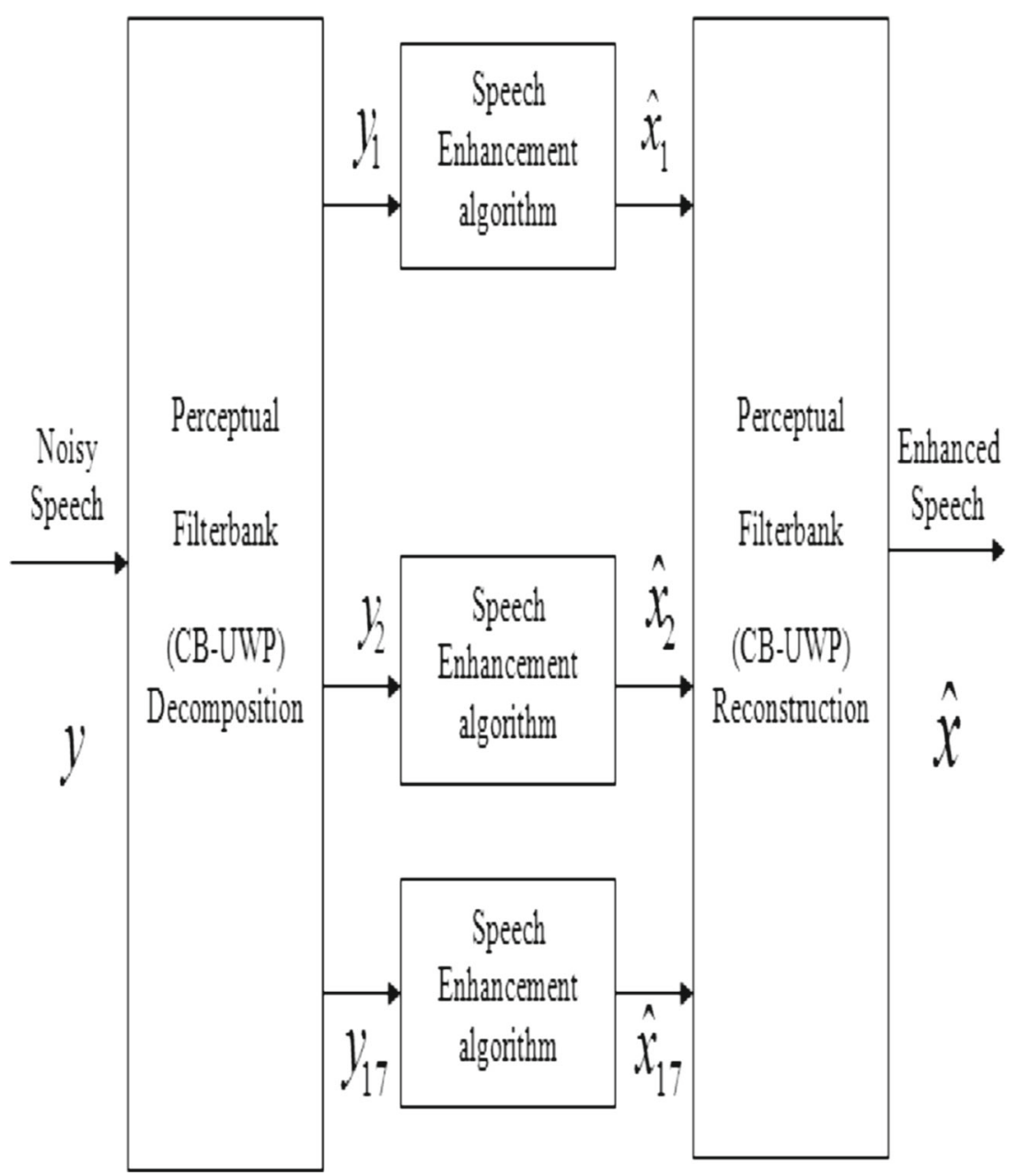

Figure 2. Block diagram of the speech enhancement system proposed by Tasmaz \& Ercelebi (2007). 


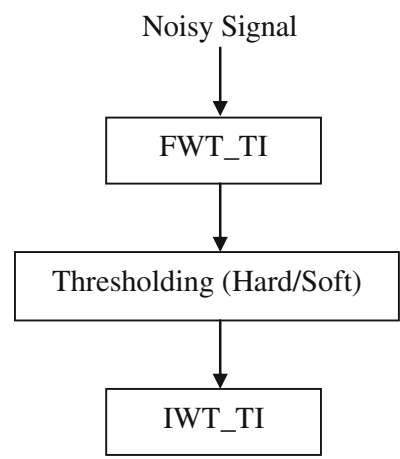

Figure 3. De-noise De-noised signal.

Chmelka \& Kozumplík 2005) and the speech enhancement system based on un-decimated wavelet packet-perceptual filter banks and MMSE-STSA estimation proposed in (Tasmaz \& Ercelebi 2007). This speech enhancement system is summarized by the following block diagram (figure 2):

Where the Perceptual Filterbank (CB-UWP) Decomposion of the noisy speech signal $y$ is performed by the application of the undecimated wavelet packet-perceptual transform. This decomposition leads to 17 critical sub-bands, $y_{1}, y_{2}, \ldots, y_{17}$, and this by referring to psychoacoustic model (Tasmaz \& Ercelebi 2007). Each of these critical sub-bands is enhanced by the speech enhancement algorithm proposed by Ephraim \& Malah (1984). The enhanced speech signal, $\hat{x}$ is obtained by perceptual filterbank (CB-UWP) reconstruction from the enhanced subbands $\hat{x}_{1}, \hat{x}_{2}, \ldots, \hat{x}_{17}$. Therefore for de-noising speech signal, Tasmaz \& Ercelebi (2007) have processed each noisy critical sub-band $y_{i}, 1 \leq i \leq 17$ independently as a noisy signal and denoise de-noised it by the algorithm proposed by Ephraim \& Malah (1984). This principle of signal de-noising is used in the ECG de-noising technique proposed in this work (figure 1). So each of the noisy bionic wavelet coefficients obtained from the application of the BWT to the noisy ECG signal, is processed as a noisy signal and the algorithm of signal de-noising is applied to it separately. As previously mentioned, this de-noising algorithm is based on thresholding in FWT_TI domain and can be summarized by the following block diagram given in figure 3 .

Table 1. MSE variation vs SNR.

\begin{tabular}{lcc}
\hline & \multicolumn{2}{c}{ MSE } \\
\cline { 2 - 3 } $\begin{array}{c}\text { The proposed } \\
\text { technique } \\
\text { with hard } \\
\text { thresholding }\end{array}$ & $\begin{array}{c}\text { Hard } \\
\text { thresholding } \\
\text { method based } \\
\text { on FWT_TI }\end{array}$ \\
\hline-5 & 0.0046 & 0.0066 \\
0 & 0.0014 & 0.0016 \\
5 & $4.9360 \mathrm{e}-004$ & $5.7111 \mathrm{e}-004$ \\
10 & $2.0812 \mathrm{e}-004$ & $2.4266 \mathrm{e}-004$ \\
15 & $9.4467 \mathrm{e}-005$ & $1.0752 \mathrm{e}-004$ \\
20 & $4.5579 \mathrm{e}-005$ & $5.1128 \mathrm{e}-005$ \\
\hline
\end{tabular}


Table 2. MSE variation vs SNR.

\begin{tabular}{lcc}
\hline & \multicolumn{2}{c}{ MSE } \\
\cline { 2 - 3 } $\begin{array}{c}\text { The proposed } \\
\text { technique } \\
\text { with soft } \\
\text { thresholding }\end{array}$ & $\begin{array}{c}\text { Soft } \\
\text { thresholding } \\
\text { method based } \\
\text { on FWT_TI }\end{array}$ \\
\hline-5 & 0.0141 & 0.0286 \\
0 & 0.0041 & 0.0064 \\
5 & 0.0013 & 0.0016 \\
10 & $5.0065 \mathrm{e}-004$ & $5.8519 \mathrm{e}-004$ \\
15 & $2.2111 \mathrm{e}-004$ & $2.5311 \mathrm{e}-004$ \\
20 & $1.0556 \mathrm{e}-004$ & $1.1601 \mathrm{e}-004$ \\
\hline
\end{tabular}

In the proposed ECG de-noising technique, we have in first stage applied the BWT to the noisy ECG signal because there has been some outstanding features with the BWT such as nonlinearity, high sensitivity and frequency selectivity, concentrated energy distribution and its

Table 3. The techniques performance on MIT-BIH arrhythmia database.

\begin{tabular}{lcc}
\hline & \multicolumn{2}{c}{ SNR improvement (dB) } \\
\cline { 2 - 3 } Signal & $\begin{array}{c}\text { The proposed } \\
\text { technique }\end{array}$ & $\begin{array}{c}\text { The technique } \\
\text { of Sayadi \& Shamsollahi (2006) }\end{array}$ \\
\hline 100 & 7.83 & 7.8 \\
101 & 8.79 & 6.9 \\
103 & 8.63 & 7.7 \\
105 & 8.59 & 8.1 \\
112 & 7.45 & 8.2 \\
113 & 10.09 & 7.8 \\
115 & 9.79 & 7.9 \\
116 & 8.82 & 8.0 \\
117 & 8.84 & 7.9 \\
119 & 8.81 & 7.6 \\
122 & 8.32 & 6.9 \\
123 & 10.09 & 7.8 \\
200 & 9.02 & 6.9 \\
201 & 8.65 & 7.5 \\
202 & 9.76 & 7.8 \\
205 & 8.29 & 7.4 \\
209 & 7.96 & 8.1 \\
210 & 8.92 & 6.9 \\
212 & 7.69 & 6.7 \\
213 & 7.64 & 8.2 \\
219 & 8.71 & 8.0 \\
220 & 9.74 & 8.1 \\
221 & 9.59 & 7.9 \\
230 & 8.58 & 7.9 \\
233 & 8.67 & 7.8 \\
\hline & &
\end{tabular}


aptitude for signal reconstruction via inverse transform but the most distinguishing characteristic of BWT is that its resolution in the time-frequency domain can be adaptively adjusted not only by the signal frequency but also by the signal instantaneous amplitude and its first-order differential (Sayadi \& Shamsollahi 2006). Moreover this transform among wavelet transforms, tends to uncorrelated data (Bahoura \& Rouat 2006) and this can make easier the noise cancellation from the noisy ECG signal. Concerning the use of the de-noising algorithm based on thresholding in FWT_TI domain (figure 3) for de-noising the noisy bionic wavelet coefficients, this can introduce more and more adaptation for noise estimation. Moreover, the translation-invariant de-noising permits to have bionic wavelet coefficients far weaker Gibbs phenomena than thresholding based de-noising using the classical orthogonal wavelet transform. All these facts permit therefore to have a de-noise de-noised ECG signal with good quality.
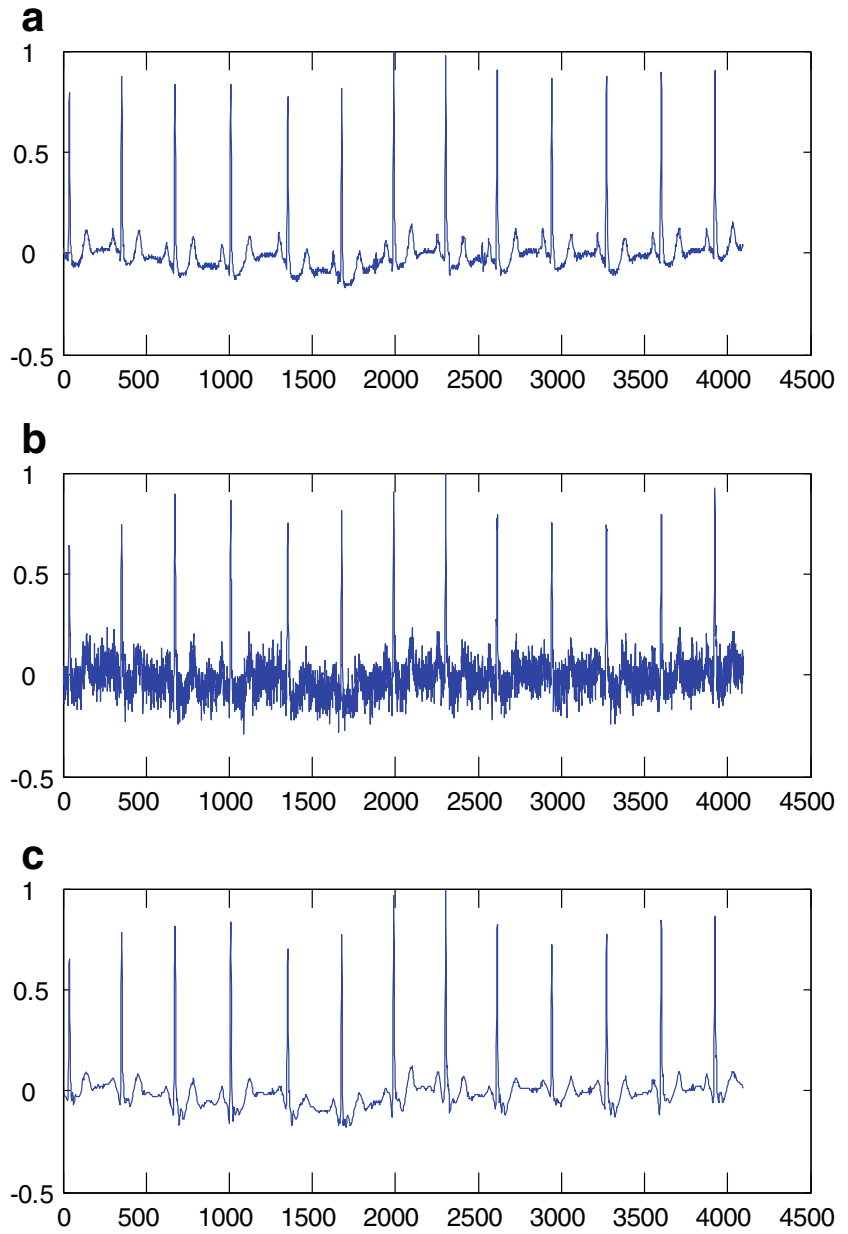

Figure 4. ECG signal corrupted by white noise with $\mathrm{SNR}=5 \mathrm{~dB}$. 


\section{Resuts and discussion}

In this work, the used ECG signals are taken from MIT-BIH database. For evaluating the performance of the proposed de-noising technique, we have used the SNR improvement measure:

$$
\begin{aligned}
\operatorname{imp}[d B] & =S N R_{\text {output }}-S N R_{\text {input }} \\
& =10 \cdot \log \left[\frac{\sum_{i}\left|s_{d}(i)-s(i)\right|^{2}}{\sum_{i}\left|s(i)-s_{n}(i)\right|^{2}}\right],
\end{aligned}
$$

where $s, s_{n}$ and $s_{d}$ are respectively the clean, the noisy and the de-noise de-noised signals.

In this paper, we have treated the case of additive Gaussian white noise, which is corrupting in artificial manner, the ECG signals with different values of SNR. In our evaluation, we have also used the mean square error (MSE) which is expressed as follows:

$$
M S E=E\left[(s(n)-\tilde{s}(n))^{2}\right]=\frac{1}{N} \sum_{n=0}^{N-1}(s(n)-\tilde{s}(n))^{2},
$$

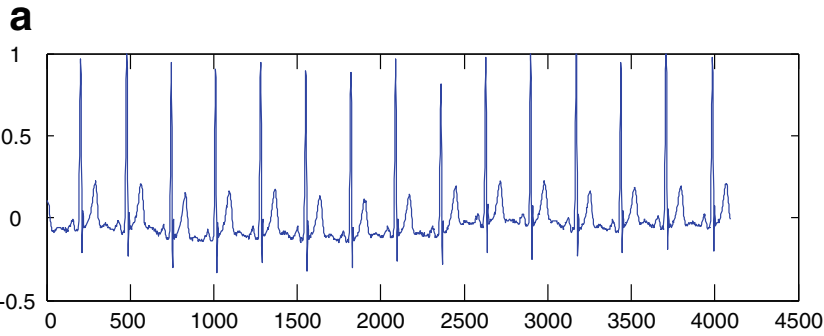

b
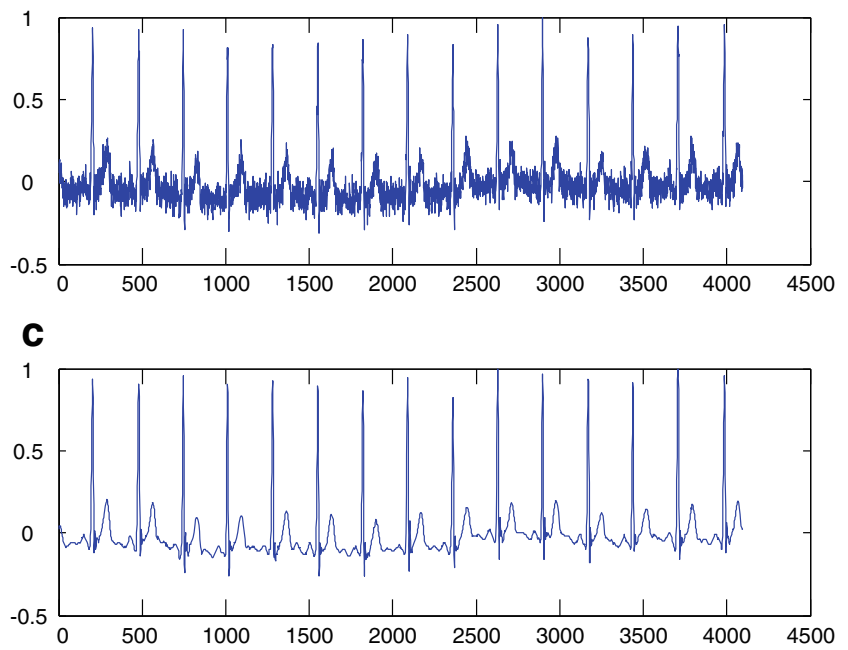

Figure 5. ECG signal corrupted by white noise with $\mathrm{SNR}=10 \mathrm{~dB}$. 
where $s(n)$ and $\tilde{s}(n)$ are respectively the clean and the de-noised signals.

Tables 1 and 2 report the obtained results from the mean square error (MSE) computation and this by applying the two de-noising techniques (the proposed technique and the conventional technique based on thresholding in FWT_TI domain) using hard or soft thresholding function.

These MSE values show that the proposed technique is better than the thresholding technique in the FWT_TI domain.

We also make a comparison between the proposed technique and the technique of Sayadi \& Shamsollahi (2006). The latter is based on thresholding of the noisy bionic wavelet coefficients obtained from the application of the BWT to the noisy ECG signal. For thresholds computation, Sayadi \& Shamsollahi (2006) have used the T-function as follow:

$$
t h r=\frac{\sigma}{\sum_{i} \alpha_{i} T_{f_{s}}(i)} \sqrt{2 \cdot \log _{2}(N)},
$$

where $t h r$ is the threshold value, $N$ is the length of the noisy signal and $\sigma=A M F S / 0.6745$ where $A M F S$ is the absolute median estimated on the first scale of the noisy bionic wavelet coefficients. The factor $T_{f_{s}}(i)$ is the value of the T-function in the first step of computing.
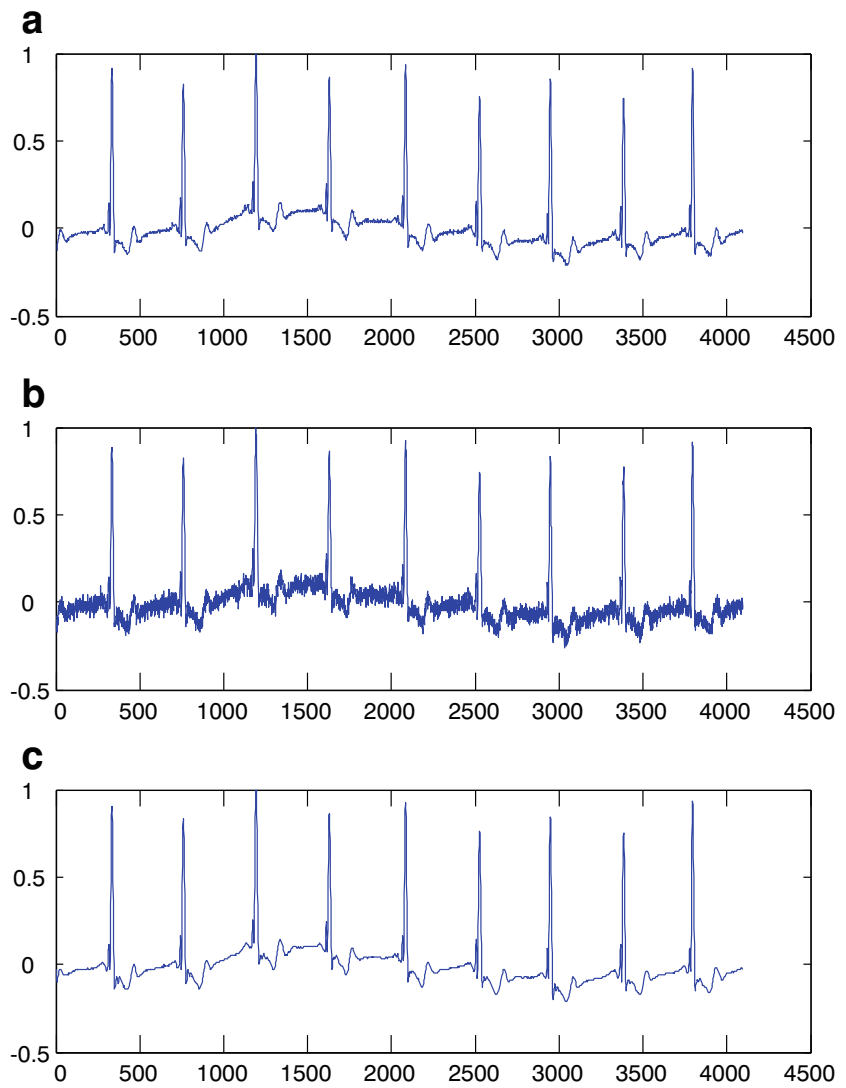

Figure 6. ECG signal corrupted by white noise with $\mathrm{SNR}=15 \mathrm{~dB}$. 
In fact, a weighted average of the values of the T-function in the first scale of decomposition with the bionic wavelet transform, was added to the following formula:

$$
\text { thr }=\sigma \cdot \sqrt{2 \cdot \log _{2}(N)} .
$$

The $\alpha_{i}$-weights are selected with try and error for the algorithm to have the highest performance (Sayadi \& Shamsollahi 2006). However, an experimentally right choice is to let the $\alpha_{i}$ coefficients be a decreasing function (Sayadi \& Shamsollahi 2006).

The proposed technique and the technique of Sayadi \& Shamsollahi (2006) are also applied to a large number of ECG signals taken from MIT-BIH and corrupted by a Gaussian white noise with signal to noise ratio (SNR) equals to $5 \mathrm{~dB}$. The obtained results from SNR improvement are reported in table 3 .

These obtained results (table 3) show clearly that the proposed technique outperforms the technique of Sayadi \& Shamsollahi (2006).

Figures 4, 5 and 6 show some examples of ECG de-noising using the proposed technique. Figure 7 illustrates the curves of variation of SNR improvement vs input SNR. This curves are obtained from the application of the proposed technique and the conventional technique.

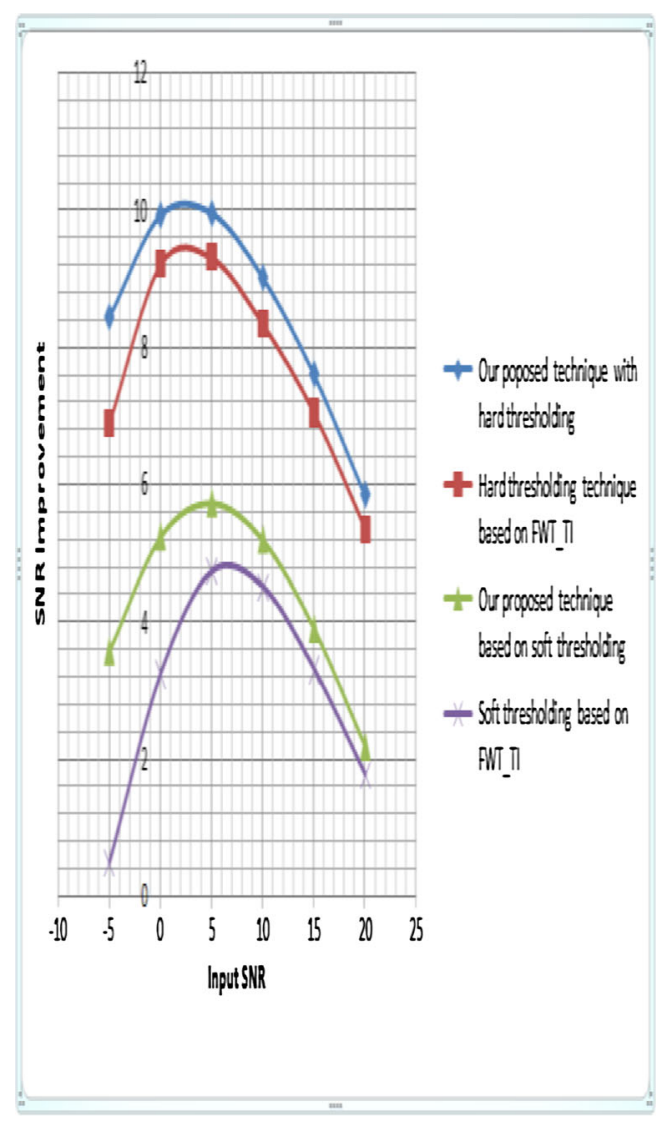

Figure 7. Improvement vs. input SNR for our proposed method and thresholding method based on FWT_TI. 
As shown in figure 7, we can remark that the proposed de-noising technique outperforms the second technique based on thresholding in FWT_TI domain. This figure also shows that when using Hard Thresholding, the obtained results are better than those obtained when using Soft Thresholding and this for the two techniques. We can also remark that in case of hard thresholding, the maximum of SNR improvement is about $10 \mathrm{~dB}$ when using the proposed technique and is about $9.5 \mathrm{~dB}$ when using the thresholding technique in the FWT_TI domain. In case of Soft thresholding, this maximum is about $5.6 \mathrm{~dB}$ when using the proposed de-noising technique and is about $4.8 \mathrm{~dB}$ when using the second technique.

\section{Conclusion}

In this paper, we have proposed a new de-noising technique based on the thresholding of the coefficients obtained from the application of the forward wavelet transform translation invariant (FWT_TI) in the bionic wavelet transform domain. The FWT_TI application is performed to each bionic wavelet coefficient obtained from the application of the BWT to the noisy ECG signal. The results obtained from SNR and MSE computations show that the proposed technique outperforms the technique based on thresholding in FWT_TI domain and the thresholding technique in the BWT domain.

\section{References}

Addison P S 2005 Wavelet transforms and the ECG a review. Institute of physics publishing, pp 155-199

Bahoura M and Rouat J 2006 Wavelet speech enhancement based on time-scale adaptation. Speech Commun. 48(12): 1620-1637

Berger J, Coifman R R and Goldberg M 1994 Removing noise from music using local trigonometric bases and wavelet packets. J. Audio Eng. Soc. 42

Chmelka L and Kozumplík J 2005 Wavelet-based wiener filter for electrocardiogram signal denoising. Comput. Cardiol. 32: 771-774

Coifman R R and Donoho D L 1995 Translation-Invariant De-noising. In A. Antoniadis and G. Oppenheim (eds.), Wavelets and Statistics, pp 125-150, Springer-Verlag

Ephraim Y and Malah D 1984 Speech enhancement using a minimum mean square error short time spectral amplitude estimator. IEEE Trans. Acoust. Speech Signal Process. ASSP-32(6): 1109-1121

Gao W, Li H, Zhang Z and Wang T 2003 De-noising of ECG signal based on stationary wavelet transform. Acta Electr. Sin. 32: 238-240

Han J Y, Lee S K and Park H B 2009 Denoising ECG using Translation Invariant Multiwavelet. Int. J. Electr. Comput. Syst. Eng. 3(3): 138-142

Janusek D, Kania M, Zaczek1 R, Zavala-Fernandez H, Zbieć A, Opolski1 G and Maniewski R 2011 Application of Wavelet Based Denoising for T-Wave Alternans Analysis in High Resolution ECG Maps. Measurement Science Review, 11(6): 181-184

Johnson M T, Yuan X and Ren Y 2007 Speech signal enhancement through adaptive wavelet thresholding. Sci. Direct. Speech Commun. 49: 123-133

Mallat S and Zhong S 1991 Charactrization of signal from multiscale edges. IEEE Trans. Pattern Anal. Mach. Intell. 14: 701-732

Mourad T, Lotfi S, Sabeur A and Adnane C 2010 Recurrent neural network and bionic wavelet transform for speech enhancement. Int. J. Signal Imaging Syst. Eng. 3(2): 136-144

Mourad T, Aouinet A, Riadh B and Adnane C 2012 ECG analysis based on wavelet transform. IJCSI Int. J. Comput. Sci. Issues, 9(Issue 1, No. 3): 427-435

Nikolaev N and Gotchev A 2000 ECG signal denoising using wavelet domain wiener filtering. Proceeding of the European Association for Signal and Image Processing (EURASIP) 
Sahambi J S, Tandon S N and Bhatt R K P 1998 Wavelet based ST-segment analysis. Med. Biol. Comput. 36: $568-572$

Saito N 1994 Feature Extraction using Local Discriminant Basis. Yale Dissertation December 1994

Sayadi O and Shamsollahi M B 2006 ECG Denoising with Adaptive Bionic Wavelet Transform. Proc. EMBS, pp. 6597-6600

Tasmaz H and Ercelebi E 2007 Speech enhancement based on undecimated wavelet packet-perceptual filterbanks and MMSE-STSA estimation in various noise environments. Digital Signal Processing, (p.16, in press, available online 12 October 2007).

Yao J and Zhang Y T 1999 Cochlear is an inhomogeneous, active and nonlinear model. Proc. IEEE EMBS/BMES, Atlanta, 1031

Yao J and Zhang Y T 2000 From Otoacoustic Emission Modeling to Bionic Wavelet Transform. Proceedings of the 22nd Annual EMBS International Conference, 314-316

Yao J and Zhang Y T 2001 Bionic wavelet transform: a new time-frequency method based on an auditory model. IEEE Trans. Biomed. Eng. 48(8): 856-863

Yuan X 2003 Auditory Model-based Bionic Wavelet Transform for Speech Enhancement. M.Sc. Thesis, Marquette University, Speech and Signal Processing Lab Milwaukee, Wisconsin 